\title{
Spatio-temporal Change Detection of Urban Heat Islands Using Spatial Interpolation
}

\author{
Shrutilipi Bhattacharjee ${ }^{1}$, Titas Aikat ${ }^{2}$, and Soumya K. Ghosh ${ }^{3}$ \\ School of Information Technology, Indian Institute of Technology Kharagpur, India \\ \{shrutilipi.2007, titasaikat\}@gmail.com, skg@iitkgp.ac.in
}

\begin{abstract}
The urban heat island has drawn significant attention in recent years. It has grabbed an unprecedented research interest in the field of remote sensing and geographic information system. This work focuses on spatial interpolation based change detection of heat islands and finding the dynamics of the heat islands. Performance checking is done on derived spatial attribute, land surface temperature (LST). The LSTs of two distinct Landsat ETM+ satellite images (of years 2000 and 2010) are used to verify the accuracy of the proposed algorithm.
\end{abstract}

Keywords: Urban Heat Island, Geographic Information System, Spatial Interpolation, Inverse Distance Weighting.

\section{Introduction}

The 20th century is witnessing "the rapid urbanization of the world's population", as the global proportion of urban population is rising dramatically [1]. It is well known and documented that urbanization can have significant effects on local weather and climate. Among these effects, one of the most important is the urban heat island (UHI), which is the direct representation of environmental degradation. An urban heat island is a geographic region which is significantly warmer than its surrounding areas. The higher urbanization leads to more distinct urban heat island with significant temperature differences between urban and rural areas.

Satellite remote sensing is widely used to detect the growth of heat islands, their spatio-temporal drifting, update existing maps, etc. In recent years, many change detection tools and techniques, such as, visual interpretation, pixel-based methods and object-based approaches etc. have been developed. The remote sensing satellite images are in the form of raster data, whereas, geographic information system (GIS) mostly deals with vector data in form of geographic markup language (GML) file, shapefile, etc. Further, GIS layers are formed by derived spatial attributes, such as, land surface temperature (LST), moisture stress index (MSI) etc. So, image analysis is not always a good choice for GIS. The proposed scheme of UHI change detection primarily focuses on the spatio-temporal drifting of the point/or the spatial region responsible for drastic change of temperature, refered as UHI, and finding the dynamics of these UHI points and their temporal path of drifting. Two derived attribute datasets can be analysed to capture

P. Maji et al. (Eds.): PReMI 2013, LNCS 8251, pp. 222-228, 2013.

(C) Springer-Verlag Berlin Heidelberg 2013 
the change from one dataset to another. The reference dataset is considered with respect to which the change detection has been carried out in the second (target) dataset. It is done through temporal mapping of the spatial interpolation technique from the reference dataset to the target dataset. In this work, LSTs, derived from Landsat ETM+ image datasets (source: USGS1 ) are used for analysis and one of the most popular interpolation techniques, inverse distance weighting $(I D W)[2$, is considered for detecting drift of UHI points.

UHIs can be divided into two types, based on the methods of temperature measurements, namely, the canopy layer heat island and the boundary layer heat island [3]. Several literature surveys have been reported regarding detailed description of UHI formation and detection, their effect analysis, strategies to reduce urban heat island etc. [4] [5. Oleson et al. [6] analysed UHI characteristics in details with respect to a global climate model. Weng et al. [7] have studied the urban heat island (UHI) phenomenon to examine the spatial distribution of surface radiant temperatures in Guangzhou, China. Yuan et al. [8] investigated the relationships between the land surface temperature (LST), percent impervious surface area (ISA), and the normalized difference vegetation index (NDVI) to compare the ISA and NDVI as the influential parameter for surface UHI effects. Characteristics of UHI and boundary layer structures were analysed in [9] using conventional and Moderate Resolution Imaging Spectroradiometer (MODIS) observations, taking Beijing area, China as the example scenario case study.

The propsed work analyses the derived attribute information from satellite images for detecting spatio-temporal drifting of UHI points and finding their track of movement. The main objectives of this work are as follows,

- Study of LST for Landsat ETM+ image datasets.

- Detection of spatio-temporal drifting of UHI points from reference dataset to the target dataset.

- Analysing the temporal movement of UHI points and finding the drifting path.

The paper is organized as follows. Section 2 gives detailed description of the proposed framework. Case study with the proposed algorithm with ETM+ image datasets, is specified in the Section 3 Finally the conclusion is drawn in the Section 4

\section{Framework to Analyse UHI Drifting}

The proposed framework deals with the derived spatial attribute land surface temperature, LST, which is present in the geographic data repository in the form of vector data. Finding spatio-temporal drifting point of the heat islands in the target dataset, is based on spatial interpolation technique with respect to the reference dataset.

For this work, it is assumed that the UHI points in the reference dataset are known. LST values of those UHI points are plotted and a curve of LSTs is

\footnotetext{
${ }^{1}$ http://www.usgs.gov/
} 


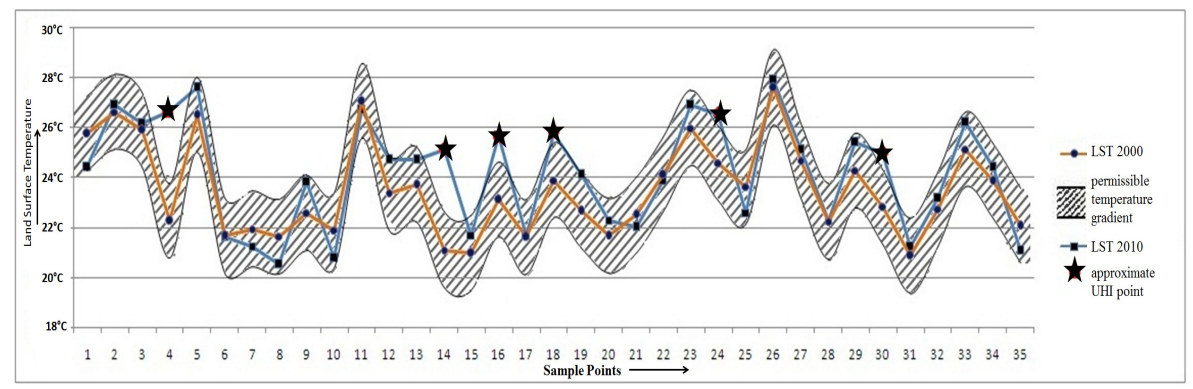

Fig. 1. LST at each UHI for the reference and the target datasets with permissible temperature gradient as buffer

obtained (refer Fig. 11). Taking the average temporal change of LST value from the reference year dataset to the target year dataset, a buffer is drawn along the curve. It is denoted as the permissible temperature gradient in Fig. 1 The target dataset where the drift of the newly developed UHI points is to be predicted, are identified which represents the same coordinate points as the reference dataset. They are considered as the candidate UHI points in the target dataset. LSTs of the target dataset at those points are also plotted accordingly. If the curve corresponding to any of the UHI point is changed more than threshold (i.e. above the permissible temperature gradient), this point is taken as the newly found candidate UHI point. It is shown in Fig. 1 where, UHI point ids: 4, 14, 16, 18,24 and 30 (given in the $\mathrm{X}$ axis, identified with the symbol $\star$ ) are selected as the candidate/ approximate UHI points in the target dataset.

The UHIs which are selected as the candidate UHI points are analysed further for drift detection. The overall framework is depicted in Fig. 2 To find the drifted UHI points in the target dataset, the selected $i^{\text {th }}$ UHI point is then represented by its surrounding points as per spatial interpolation technique, IDW. A predefined number of interpolating points are chosen against each candidate UHI point. The LST of selected UHI point is represented as the weighted sum of its surrounding interpolating points. So, $i^{\text {th }}$ UHI point in both reference and target dataset can be represented as,

$S_{1 i}=w_{1 i 1} \cdot K_{1 i 1}+w_{1 i 2} \cdot K_{1 i 2}+\ldots+w_{1 i j} \cdot K_{1 i j}$

$S_{2 i}=w_{2 i 1} \cdot K_{2 i 1}+w_{2 i 2} \cdot K_{2 i 2}+\ldots+w_{2 i j} \cdot K_{2 i j}$

where $S_{1 i}$ and $S_{2 i}$ represent $i^{t h}$ UHI point's LST of the reference and the target dataset, respectively. The $K_{m i j}$ and the $w_{m i j}$ represent LST of the $j^{\text {th }}$ interpolating point and its weight assigned by the $I D W$ with respect to $i^{\text {th }}$ UHI point, repectively. Here, $m=1$ and 2 for the reference and the target datasets, respectively. Now, as per $I D W, w_{1 i j}$ and $w_{2 i j}$ are the functions of distance and are same for any $j$. Difference in $S_{1 i}$ and $S_{2 i}$ causes due to change in LST values of its surrounding interpolating points, i.e. for the difference of $K_{1 i j}$ and $K_{2 i j}$, for all $j$.

Now, each pair of $K_{1 i j}$ and $K_{2 i j}$ for $i^{\text {th }}$ UHI point are examined to find the maximum difference above a predefined threshold. The $j^{\text {th }}$ interpolating point at which maximum change is found, is taken into consideration and analysed 


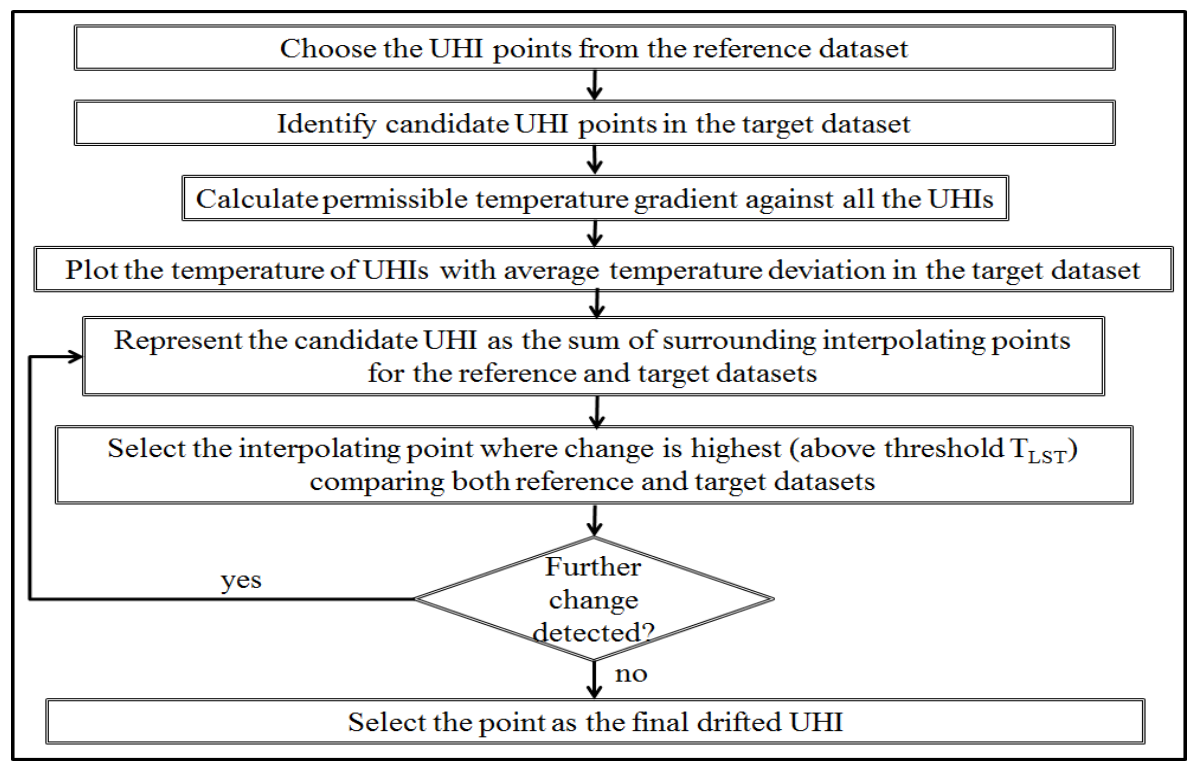

Fig. 2. Process flow to select drifted UHI point in the target dataset

further. This process iterates until no change found or after certain number of iterations are done corresponding to a particular UHI point. Finally, the selected coordinate point where the iteration stops is selected as the drifted UHI point with respect to the reference dataset. This process will also help in finding the temporal track of movement of UHI points. The selected UHI point in each of the iterations will be further connected to get the probable path of movement for the UHI points. The same process can be applied for all the candidate UHI points. As the number of points are finite, this process is also finite. The mechanism is shown in Algorithm 1

\section{Case Study}

For the performance study and result analysis, an experiment has been carried out with derived LST data of the year 2000 (reference dataset) and 2010 (target dataset) of Kolkata which is one of the major metropolitan cities in India (central coordinate: $22.567^{\circ} \mathrm{N} 88.367^{\circ} \mathrm{E}$ ). Six UHI points are taken from the reference dataset. The drifted UHI points are identified using the Algorithm 1, For example, in Fig. 3, point no. 30 is taken for further analysis using Algorithm 1. At initialization phase, it is labelled as point 30. After first iteration, newly selected candidate UHI is point no. $30_{1}$, then point no. $30_{2}$ and so on. After $3^{\text {rd }}$ iteration, no further cange is found. Point no. $30_{3}$ is selected as the final drifted UHI point corresponding to the candidate UHI no. 30. The probable path of movement of the UHI point no. 30 can be given as, $30 \rightarrow 30_{1} \rightarrow 30_{2} \rightarrow 30_{3}$ (refer Fig. 3). Though it is shown in distinct Landsat ETM+ satellite image, 
Algorithm. Find drifted UHI point from candidate UHI point

Input: Candidate UHI point $S_{2 i}$, maximum number of iterations: $n$, number of interpolation points against each candidate UHI point: $m$

Output: Drifted UHI point $A_{i}$

$1 A_{i}=S_{2 i}$

2 while number of iterations for $A_{i}<n$ do

$3|\max =| S_{2 i}-S_{1 i} \mid$;

4 foreach $j=1 \rightarrow m$ do

$5 \quad \mid$ if $\left|K_{2 i j}-K_{1 i j}\right|>\max$ then

$\mathbf{7}$
$\mathbf{8}$$\quad \begin{aligned} & \max =\left|K_{1 i j}-K_{2 i j}\right| ; \\ & \mathrm{p}=\mathrm{j} ;\end{aligned}$

$8 \quad$ end

$9 \quad$ end

$10 \quad$ if $\max ==\left|S_{2 i}-S_{1 i}\right|$ then

$\mathbf{1 1}$
$\mathbf{1 2}$

13

14

15

; else

I $A_{i}=p^{t h}$ interpolating point of $i^{\text {th }}$ UHI point representing $S_{2 i}$ end

16 ;

17 end

18 Output $A_{i}$ as the final drifted UHI point

Algorithm 1. Detection of drifted UHI point from the candidate UHI point

the actual computation is done on derived data. Analysis of six UHI points are shown in Table 1 . The candidate and the drifted UHI point's coordinate information are recorded against each of the UHI points.

Discussion: The proposed scheme is efficient compared to pixel-by-pixel analysis. Typically pixel-by-pixel analysis is time consuming and often requirs expert's intervention. For the proposed methodology, number of pixels to be analysed and the number of iterations are less. In case of the given example, Algorithm 1 takes maximum 10 iterations for the candidate UHI point detection. Though raw Landsat image is not required for drifted UHI point detection through proposed scheme, for checking the accuracy of this scheme, two images and their corresponding derived attribute information are taken for the case study.

\section{Conclusion}

The urban heat islands are becoming a major threat in today's world. It has adverse effect in terms of global warming, air pollution, human health, etc. The proposed framework focuses on spatio-temporal drifting of urban heat islands. It may be useful to find the pattern of movement of UHIs to take necessary actions. This methodology is found to be efficient with a very few number of sample points. The probable path of temporal drifting of UHI points can be detected by the proposed approach. This work can be extended further for investigating the 


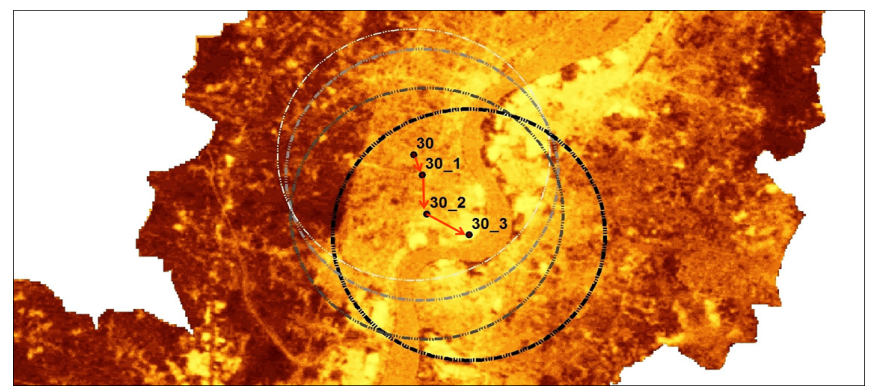

Fig. 3. Drifting of a UHI point from its candidate UHI location

Table 1. Experimentation details using Algorithm 1 on Landsat ETM+ images

\begin{tabular}{|c|c|c|c|}
\hline $\begin{array}{c}\text { Candidate } \\
\text { UHI Point } \\
\text { ID }\end{array}$ & $\begin{array}{c}\text { Candidate UHI } \\
\text { Coordinate Location }\end{array}$ & $\begin{array}{c}\text { Final Drifted UHI } \\
\text { Coordinate Location }\end{array}$ & $\begin{array}{c}\text { Temperature } \\
\text { Difference } \\
\left({ }^{\circ} \mathbf{C}\right)\end{array}$ \\
\hline 1 & $\left(22.5686^{\circ} \mathrm{N}, 88.1824^{\circ} \mathrm{E}\right)$ & $\left(22.5733^{\circ} \mathrm{N}, 88.1772^{\circ} \mathrm{E}\right)$ & 11.96 \\
\hline 2 & $\left(22.8064^{\circ} \mathrm{N}, 88.3173^{\circ} \mathrm{E}\right)$ & $\left(20.8057^{\circ} \mathrm{N}, 88.3120^{\circ} \mathrm{E}\right)$ & 11.66 \\
\hline 3 & $\left(22.9686^{\circ} \mathrm{N}, 88.4591^{\circ} \mathrm{E}\right)$ & $\left(22.9585^{\circ} \mathrm{N}, 88.4652^{\circ} \mathrm{E}\right)$ & 9.59 \\
\hline 4 & $\left(22.8881^{\circ} \mathrm{N}, 88.3970^{\circ} \mathrm{E}\right)$ & $\left(22.8826^{\circ} \mathrm{N}, 88.4128^{\circ} \mathrm{E}\right)$ & 9.09 \\
\hline 5 & $\left(22.5939^{\circ} \mathrm{N}, 88.3213^{\circ} \mathrm{E}\right)$ & $\left(22.6013^{\circ} \mathrm{N}, 88.3079^{\circ} \mathrm{E}\right)$ & 7.60 \\
\hline 6 & $\left(22.8497^{\circ} \mathrm{N}, 88.3586^{\circ} \mathrm{E}\right)$ & $\left(22.8323^{\circ} \mathrm{N}, 88.3703^{\circ} \mathrm{E}\right)$ & 7.75 \\
\hline
\end{tabular}

drifting of UHI points with the growth of urbanizations, abatement of vegetation cover etc.

\section{References}

1. United Nations World urbanization prospects: The 2005 revision (2006)

2. Li, J., Heap, A.D.: A review of comparative studies of spatial interpolation methods in environmental sciences: Performance and impact factors. Ecological Informatics 6(3), 228-241 (2011)

3. Hekster, F., De Voogt, P., Pijinenburg, A., Laane, R.: Perfluoroalkylated substances: Aquatic environmental assessment. Rijksinstituut voor Kust en Zee/RIKZ 99 (2002)

4. Rizwan, A.M., Dennis, L.Y., Liu, C.: A review on the generation, determination and mitigation of urban heat island. Journal of Environmental Sciences 20(1), 120-128 (2008)

5. US EPA: Draft Report: In reducing urban heat islands: Compendium of strategies. Urban Heat Island basics (2003)

6. Oleson, K.W., Bonan, G.B., Feddema, J., Jackson, T.: An examination of urban heat island characteristics in a global climate model. International Journal of Climatology 31(12), 1848-1865 (2011) 
7. Weng, Q.: Fractal analysis of satellite-detected urban heat island effect. Photogrammetric Engineering and Remote Sensing 69(5), 555-566 (2003)

8. Yuan, F., Bauer, M.E.: Comparison of impervious surface area and normalized difference vegetation index as indicators of surface urban heat island effects in landsat imagery. Remote Sensing of Environment 106(3), 375-386 (2007)

9. Miao, S., Chen, F., LeMone, M.A., Tewari, M., Li, Q., Wang, Y.: An observational and modeling study of characteristics of urban heat island and boundary layer structures in beijing. Journal of Applied Meteorology and Climatology 48(3), 484-501 (2009) 